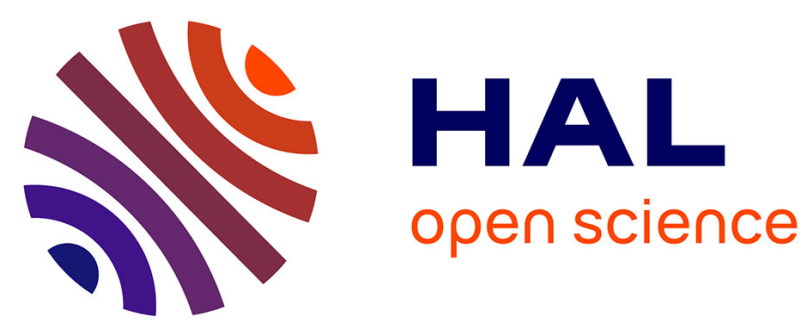

\title{
Fissuration polymodale dans les matériaux viscoélastiques orthotropes
}

Rostand Moutou Pitti, Frédéric Dubois

\section{To cite this version:}

Rostand Moutou Pitti, Frédéric Dubois. Fissuration polymodale dans les matériaux viscoélastiques orthotropes. Comptes Rendus Mécanique, 2009, 337 (11-12), pp.748-754. 10.1016/j.crme.2009.10.008 . hal-00639260

\section{HAL Id: hal-00639260 \\ https://hal-unilim.archives-ouvertes.fr/hal-00639260}

Submitted on 11 Mar 2019

HAL is a multi-disciplinary open access archive for the deposit and dissemination of scientific research documents, whether they are published or not. The documents may come from teaching and research institutions in France or abroad, or from public or private research centers.
L'archive ouverte pluridisciplinaire HAL, est destinée au dépôt et à la diffusion de documents scientifiques de niveau recherche, publiés ou non, émanant des établissements d'enseignement et de recherche français ou étrangers, des laboratoires publics ou privés.

\section{다)(1) $(5$}

Distributed under a Creative Commons Attribution - NonCommerciall 4.0 International 


\title{
Fissuration polymodale dans les matériaux viscoélastiques orthotropes
}

\author{
Rostand Moutou Pitti ${ }^{\mathrm{a}, *}$, Frédéric Dubois ${ }^{\mathrm{b}}$ \\ a LAMI (Laboratoire de mécanique et ingénierie), polytech' Clermont Ferrand, université Blaise-Pascals, BP 206, 63174 Aubière cedex, France \\ ${ }^{\mathrm{b}}$ Université de Limoges, groupe d'étude des matériaux hétérogènes, équipe génie civil \& durabilité, centre universitaire de Génie Civil, \\ 19300 Egletons, France
}

Reçu le 20 juin 2009 ; accepté après révision le 22 octobre 2009

Disponible sur Internet le 3 novembre 2009

\section{Résumé}

Ce travail présente un algorithme numérique de l'amorçage et de la propagation de fissure en mode mixte dans un milieu viscoélastique orthotrope. Le modèle numérique couple la résolution par éléments finis du comportement viscoélastique au calcul de l'intégrale $M \theta$ permettant le découplage des modes de rupture en termes de facteur d'intensité de contrainte et de taux de restitution d'énergie. L'application proposée utilise une éprouvette $2 \mathrm{MCG}$ permettant conjointement l'obtention des différents taux de mixités et une stabilité de la propagation de fissure. L'algorithme éléments finis permet de modéliser la propagation du front de fissure en simulant la décohésion progressive des lèvres dans la zone d'élaboration. L'ensemble est piloté par une fonction de charge intégrant les performances énergétiques à la rupture des différents modes (I et II) en configuration plane. La zone d'élaboration est définie via un champ de contrainte en mode mixte défini dans la zone singulière. Pour citer cet article: R. Moutou Pitti, F. Dubois, C. R. Mecanique 337 (2009).

(C) 2009 Académie des sciences. Publié par Elsevier Masson SAS. Tous droits réservés.

\section{Abstract}

Polimodal fracture in orthotropic viscoelastic media. This Note deals with an algorithmic approach about the crack initiation and the crack growth in a viscoelastic media for mixed mode configurations. This numerical model couples a finite element resolution of viscoelastic behavior and the $M \theta$ integral calculus allowing a mixed mode separation in terms of stress intensity factors and energy release rate. The numerical application uses a $2 \mathrm{MCG}$ specimen allowing, in the same time, different mixed mode ratios and a crack growth stability. The finite element algorithm allows us to model the crack tip advance by taking into account the crack lip uncohesion in the process zone. It size is defined by taking into account stress field in the crack tip vicinity. To cite this article: R. Moutou Pitti, F. Dubois, C. R. Mecanique 337 (2009).

(C) 2009 Académie des sciences. Publié par Elsevier Masson SAS. Tous droits réservés.

Mots-clés : Rupture; Modes mixtes ; Intégrales indépendantes ; Propagation de fissure

Keywords: Rupture; Mixed modes; Path-independent integrals; Crack growth process

\footnotetext{
* Auteur correspondant.

Adresse e-mail : Rostand.moutou.pitti@polytech.univ-bpclermont.fr (R. Moutou Pitti).
} 


\section{Abridged English version}

The important use of polymers or composites in engineering structures required the best understanding of their fracture mechanical behavior. Also, these materials are often characterized by a viscoelastic response and orthotropic or anisotropic characters. In the most cases, these structures are submitted to complex solicitations modifying their mechanical properties and leading to the important damaging and the local micro-crack. In the durability context, it appears very important to drive the evolution speed of the different defaults. That way, new numerical and experimental approaches enable us to understand the crack initiation and the crack growth process in viscoelastic orthotropic materials is required. This work is based on the coupling of the non-path-dependent integral $M \theta$ generalized for viscoelastic media and crack growth process [3], Eq. (1) and a complex finite element algorithm about the crack growth process in time-dependent material, Fig. 2. This work allows us to compute the crack growth process by taking into account the time uncohesion of the crack lips in the process zone. The time step advance is calculated according to a dichotomy algorithm. Its criterion is based on the use of a functional formed by critical time values of energy release rate separated in terms of opening mode and shear mode parts, Eq. (5). A numerical application is based on a creep test using a timber mixed mode crack growth specimen. Results put in evidence the algorithm capacities to separate fracture mode during the crack growth and to compute the crack tip speed versus time. This model can be employed for other complex loadings like ramp tests and fatigue in force or displacements under different loading speeds.

\section{Introduction}

La problématique de la durée de vie des structures et de leur réparation nécessite d'introduire dans les codes de calcul des lois de comportement héréditaires caractérisées par des dépendances temporelles. La conception de structures intelligentes emploie de plus en plus des matériaux possédant des directions privilégiées. Ainsi, l'ingénieur est confronté à des matériaux souvent orthotropes et possédant des comportements linéaires mais viscoélastiques. Or, la maîtrise des directions privilégiées induit, d'un autre côté, des directions de faiblesse dans lesquelles vont se développer de l'endommagement ou de la fissuration. Dans ce dernier cas, l'étude de la durée de vie des structures impose une maîtrise des cinétiques de fissuration, tant au niveau de l'amorçage que de la propagation de ces fissures. Aujourd'hui, des modèles phénoménologiques sont développés afin de prédire l'avancée de fissure dans un matériau viscoélastique [1]. Ces modèles, adaptés en modes pures, reposent sur une modélisation simplifiée du phénomène de cohésion du front de fissure propre aux matériaux quasi-fragiles [2,3]. Ces approches, si elles donnent des résultats satisfaisants, s'appuient sur des formalismes, d'une part, d'équivalence élastique non appropriée à des considérations énergétiques ; et sur des lois de décohésions callées en élasticité et difficilement transposables au comportement viscoélastique qui est le siège de relaxations localisées en pointe de fissure et dont l'histoire est perturbée par l'évolution géométrique du milieu induite par l'avancée du front de fissure. Egalement, les approches élastiques traitant de la zone cohésive peuvent difficilement être utilisées car les aspects temporels et héréditaires caractérisant la viscoélasticité complexifient le bilan énergétique de la problématique.

Nous proposons, dans cet article, un algorithme numérique permettant d'étudier l'amorçage et la propagation de fissure dans un matériau viscoélastique orthotrope. L'originalité de cette approche consiste à raisonner dans le domaine temporel. Le modèle prend en compte une évolution du contour géométrique par remaillage localisé ou total de la géométrie. Un algorithme spécifique permet une transmission de l'histoire des champs mécaniques d'un état avant à un état après propagation. Cette transition, couplée au comportement viscoélastique, permet de traduire une décohésion progressive et temporelle des lèvres de fissure dans la zone de propagation. Cet algorithme utilise un couplage d'une modélisation incrémentale par éléments finis du comportement viscoélastique orthotrope et de l'intégrale $M \theta$ permettant de déterminer la part du taux de restitution d'énergie pour chaque mode de rupture [4,5] dans des configurations mixtes de chargement. Ce couplage fait l'objet d'une première partie. La seconde partie présente l'algorithme développé. Il intègre, à la fois, la problématique de la propagation du front de fissure, le calcul de la zone d'élaboration en mode mixte, et un schéma implicite du calcul des temps de propagation. Enfin, une dernière partie présente une application numérique. Le support géométrique utilisé est l'éprouvette 2MCG (Mixed Mode Crack Growth) [6,7]. 


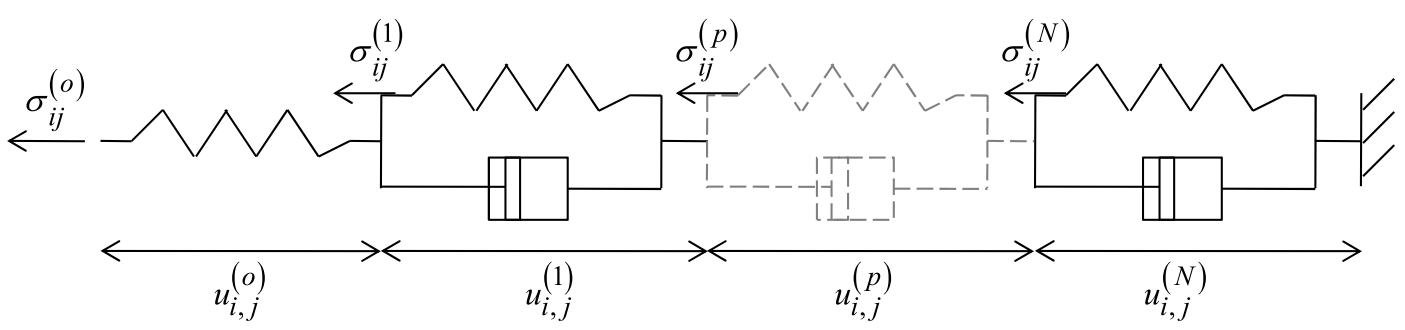

Fig. 1. Modèle de Kelvin Voigt généralisé.

Fig. 1. Generalized Kelvin Voigt model.

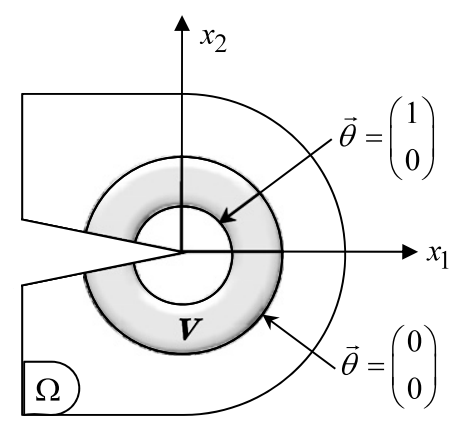

Fig. 2. Domaine d'intégration.

Fig. 2. Integration domain.

\section{Intégral $M \theta_{v}$ en propagation}

Sur la base des travaux de Chen et Shield [8], Moutou Pitti et al. [4] ont proposé une généralisation de l'intégrale $M$ adaptée à la propagation de fissure dans des matériaux viscoélastiques. Le comportement différé fait appel à un modèle de Kelvin Voigt Généralisé composé d'amortisseurs purement dissipatifs et des ressorts stockant l'énergie libre fournie par le chargement extérieur, Fig. 1. Si, initialement, ce modèle rhéologique était utilisé dans un but de compréhension des phénomènes observés, il est utilisé dans ce travail comme étant la base thermodynamique de notre approche de la mécanique de la rupture.

Ce modèle est également à la base de la modélisation du comportement viscoélastique. Dans des cas complexes comme l'orthotropie ou l'anisotropie, chaque composante du tenseur de fluage du quatrième ordre est représentée par un modèle de Kelvin Voigt généralisé. Ici, nous nous limitons à des configurations en contraintes planes. Dans ce cas, il est nécessaire d'utiliser 4 modèles spécifiques. Dans une approche énergétique, ce modèle permet d'identifier la part d'énergie libre restituable dans chaque ressort. Ainsi, l'intégrale $M \theta_{v}$ est définie par sa partition induite par chaque $p^{i \text { ème }}$ ressort :

$$
\begin{aligned}
M \theta_{v}^{(p)}= & \frac{1}{2} \cdot \int_{\Omega}\left(\sigma_{i j}^{(p)}(u) \cdot v_{i, k}^{(p)}-\sigma_{i j, k}^{(p)}(v) \cdot u_{i}^{(p)}\right) \cdot \theta_{k, j} \mathrm{~d} \Omega \\
& +\frac{1}{2} \cdot \int_{\Omega}\left(\begin{array}{c}
\left(\sigma_{i j}^{(p)}(v) \cdot\left(u_{i, j}^{(p)}\right)_{, k}+\sigma_{i j}^{(p)}(u) \cdot\left(v_{i, j}^{(p)}\right)_{, k}\right) \\
-\left(\left(\sigma_{i j}^{(p)}(v) \cdot\left(u_{i, j}^{(p)}\right)\right)_{, k}+\left(\sigma_{i j}^{(p)}(u) \cdot\left(v_{i, j}^{(p)}\right)\right)_{, k}\right)
\end{array}\right) \cdot \theta_{k} \mathrm{~d} \Omega \quad \text { avec } p \in\{0,1, \ldots, N\}
\end{aligned}
$$

Cette intégrale est caractérisée par un domaine d'intégration surfacique sous la forme d'une couronne $\Omega$ définie artificiellement par le champ $\vec{\theta}$ initialement développé par Destuynder [9]. Ce champ doit être de classe $\mathrm{C} 1$ dont les limites sont présentées en Fig. 2.

$\sigma_{i j}^{(p)}(u)$ et $\sigma_{i j}^{(p)}(v)$ représentent respectivement les contraintes réelles et virtuelles dans le $p^{i e ̀ m e ~}$ ressort du modèle rhéologique dont les déplacements respectifs associés sont $u_{i}^{(p)}$ et $v_{i}^{(p)}$. Les tenseurs de contraintes virtuels et réels possèdent une forme singulière au voisinage de la pointe de fissure. Cette singularité est définie à l'aide de facteurs 


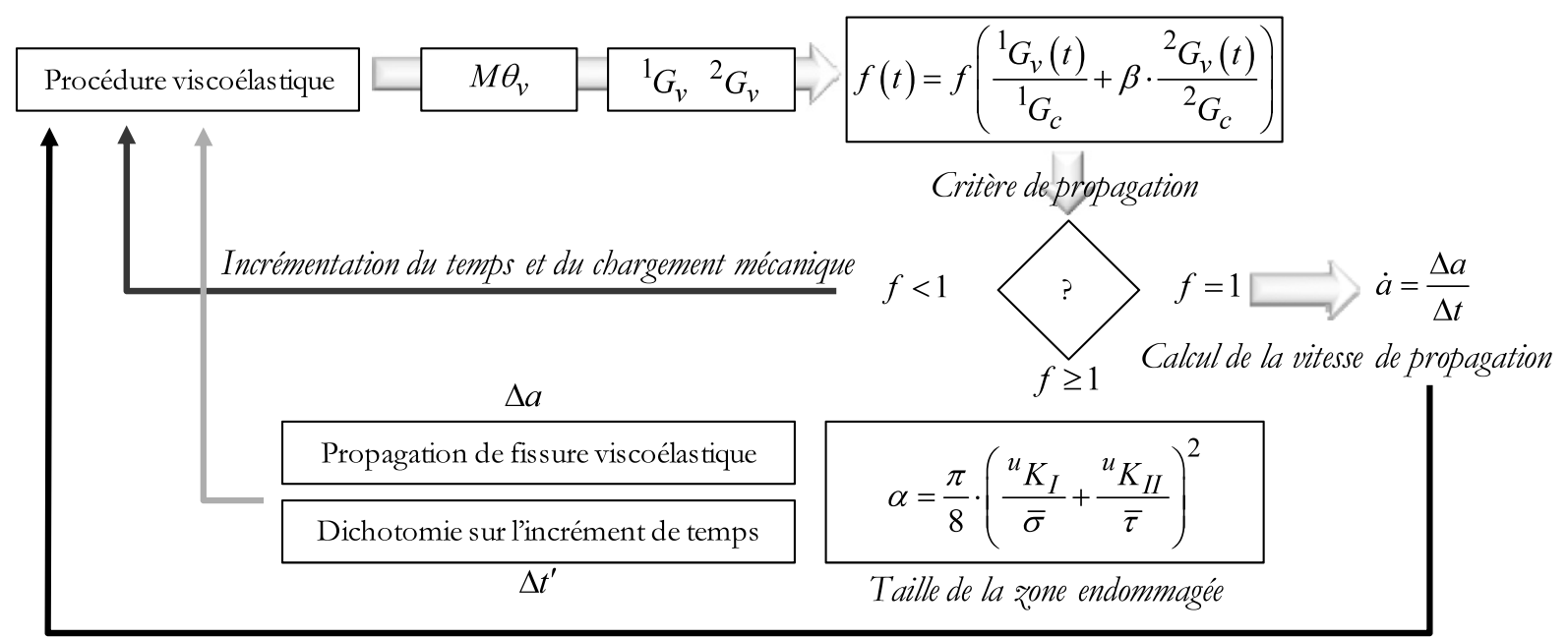

Incrémentation du chargement mécanique

Fig. 3. Algorithme de propagation.

Fig. 3. Crack growth algorithm.

d'intensité de contrainte virtuels ${ }^{v} K_{\alpha}^{(p)}$ et réels ${ }^{u} K_{\alpha}^{(p)}, \alpha$ représentant le mode de rupture considéré $(\alpha \in\{1,2\}$ pour des problèmes plans). L'utilisation de l'intégrale $M \theta_{v}$ consiste à isoler chaque mode de rupture en imposant des valeurs particulières des champs de contraintes virtuels à travers leurs facteurs d'intensité de contrainte. Ainsi, le champ de contrainte réel peut être déterminé en calculant les facteurs d'intensité de contrainte réels, et ce pour chaque mode, de la façon suivante :

$$
{ }^{u} K_{1}^{(p)}=8 \cdot \frac{M \theta_{v}^{(p)}\left({ }^{v} K_{1}^{(p)}=1,{ }^{v} K_{2}^{(p)}=0\right)}{C_{1}^{(p)}} \quad \text { et }{ }^{u} K_{2}^{(p)}=8 \cdot \frac{M \theta_{v}^{(p)}\left({ }^{v} K_{1}^{(p)}=0,{ }^{v} K_{2}^{(p)}=1\right)}{C_{2}^{(p)}}
$$

$C_{1}^{(p)}$ et $C_{2}^{(p)}$ sont les complaisances viscoélastiques associées. Les différentes partitions du taux de restitution d'énergie pour chaque mode s'expriment par :

$$
G_{v}^{(p)}={ }^{1} G_{v}^{(p)}+{ }^{2} G_{v}^{(p)} \quad \text { avec } \quad{ }^{1} G_{v}^{(p)}=C_{1}^{(p)} \cdot \frac{\left({ }^{u} K_{1}^{(p)}\right)^{2}}{8} \text { et } \quad{ }^{2} G_{v}^{(p)}=C_{2}^{(p)} \cdot \frac{\left({ }^{u} K_{2}^{(p)}\right)^{2}}{8}
$$

où ${ }^{1} G_{v}^{(p)}$ et ${ }^{2} G_{v}^{(p)}$ sont respectivement les parts de mode I et de mode II du taux de restitution d'énergie propre au $p^{i e ̀ m e}$ ressort. Finalement, le taux de restitution d'énergie, pour l'ensemble du modèle, est donné par les sommations suivantes :

$$
{ }^{1} G_{v}=\sum_{p}{ }^{1} G_{v}^{(p)} \quad \text { et } \quad{ }^{2} G_{v}=\sum_{p}{ }^{2} G_{v}^{(p)}, \quad p \in\{0,1, \ldots, N\}
$$

\section{Algorithme de propagation}

L'algorithme de propagation, Fig. 3, doit se décomposer en deux parties. La première concerne plus particulièrement l'évolution de la fissure en tant que modification du maillage éléments finis en y intégrant la problématique du comportement dépendant du temps. Cela nécessite de transporter l'histoire de l'état mécanique d'un support géométrique correspondant à l'instant avant propagation vers un autre support géométrique correspondant à l'instant suivant la propagation de fissure. Cet ensemble est regroupé dans une procédure viscoélastique. Pour des configurations en mode d'ouverture de fissure, le principe de l'algorithme est proposé par Dubois et al. [10]. Il permet de modéliser les effets de relaxation en fond de fissure incorporant une décohésion progressive des lèvres de la fissure dans la zone d'élaboration. La seconde partie concerne plus précisément des considérations de mécanique de la rupture en y intégrant la taille de la zone endommagée en pointe de fissure dans laquelle celle-ci va progresser ainsi que le critère de 

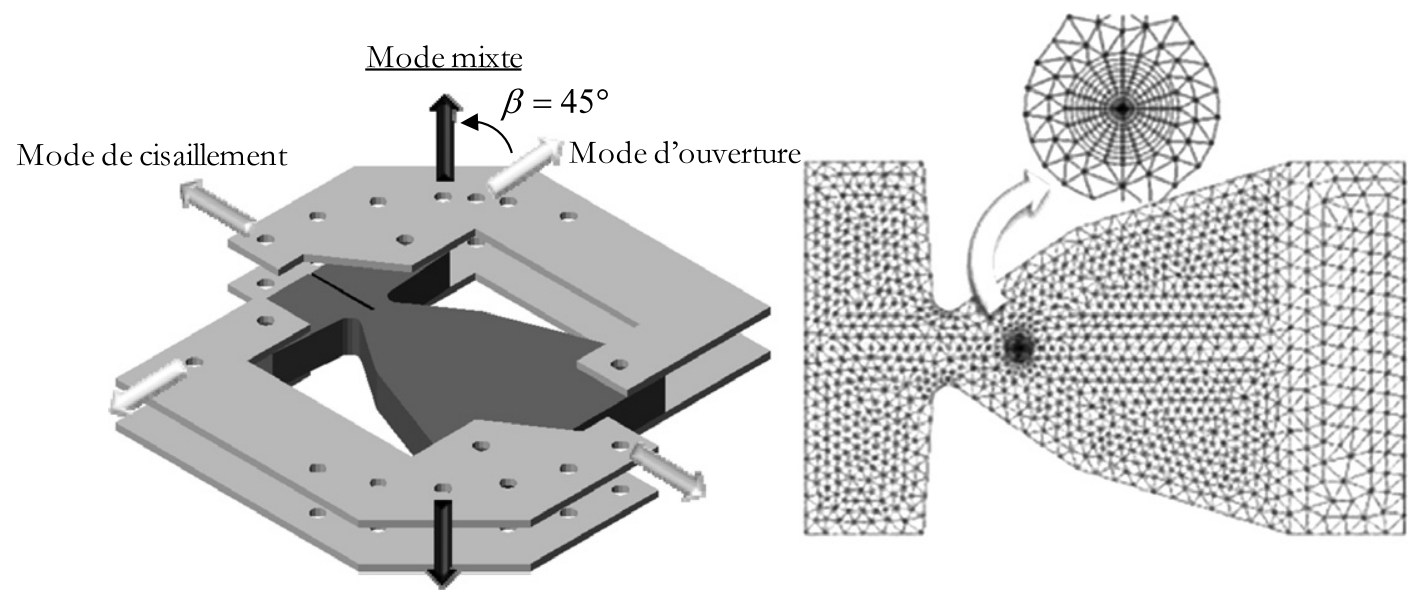

Fig. 4. Eprouvette $2 \mathrm{MCG}$ et maillage éléments finis.

Fig. 4. 2MCG specimen and FE mesh.

propagation permettant de déterminer le temps de propagation et donc la vitesse de propagation. Dans le cas d'une sollicitation en mode mixte, l'endommagement repose sur un multicritère introduit par une fonctionnelle de la résistance en traction transversale $\bar{\sigma}$ et en cisaillement $\bar{\tau}$. Enfin, l'orthotropie du matériau ne se traduit pas seulement au niveau des propriétés du tenseur de rigidité du matériau mais également en termes d'énergie de fissuration selon le sens de sollicitation. Dans ces conditions, il est classiquement utilisé une fonctionnelle notée $f$ qui est une combinaison des valeurs critiques et calculées des taux de restitution d'énergie respectivement en mode 1 et 2, Fig. 3 .

Ainsi, les critères classiques d'amorçage de fissuration se réécrivent de la façon suivante :

Fissure stationnaire ou arrêt de fissure : $f(t)<1$

Amorçage de propagation : $f(t)=1$

Instabilité finale et rupture de l'éprouvette : $f(t)>1$

Durant la phase de propagation, l'algorithme doit effectuer une dichotomie sur l'incrément de temps afin d'aboutir au respect du critère (6). Dans la modélisation de l'avancée du front de fissure, une difficulté majeure consiste à considérer, de façon conjointe, le processus de décohésion et l'effet temporel dû à la viscoélasticité. Or, les travaux de Dubois [10] ont montré que la réponse en termes de vitesse de propagation dépendait fortement de la taille de la process zone [11]. L'algorithme proposé nécessite donc de traiter, dans un incrément de temps unique, l'avancée du front de fissure dans la zone précédemment endommagée. Ceci entraîne nécessairement des hypothèses induites sur la loi de décohésion. Cependant, le modèle prend en compte un processus de décohésion progressif dans la process zone. L'hypothèse intrinsèque à la formulation incrémentale suppose que les forces de cohésion s'effacent avec une fonction temporelle linéaire, l'ensemble étant piloté par un critère énergétique dicté par la fonctionnelle $f$.

\section{Application numérique}

Cette application est basée sur un essai de fluage sur une éprouvette «Mixed Mode Crack Growth» qui se caractérise par une stabilité de la propagation de fissure en force imposée $[5,6]$ après une phase d'instabilité légère permettant un chargement à $100 \%$ de la force critique [10]. Cette phase permet, en outre, de déterminer les valeurs critiques du taux de restitution d'énergie en mode I et mode II. L'éprouvette possède deux bras en PVC permettant d'imposer différents taux de mixité dépendants de l'orientation de la sollicitation [6,7,12]. Dans cette application, l'éprouvette est sollicitée en mode mixte correspondant à une orientation angulaire de $45^{\circ}$. Le maillage éléments est présenté en Fig. 4.

Un maillage rayonnant entourant la pointe de fissure permet d'avoir une stabilité accrue du calcul de l'intégrale $M \theta_{v}$. Nous choisissons une éprouvette en pin du nord. Les propriétés en fissuration sont précisées dans le Tableau 1. Les propriétés viscoélastiques sont empruntées aux travaux de Dubois [10]. 
Tableau 1

Propriétés de rupture.

Table 1

Fracture properties.

\begin{tabular}{lllll}
\hline${ }^{1} G_{c}$ & ${ }^{2} G_{c}$ & $\beta$ & $\bar{\sigma}$ & $\bar{\tau}$ \\
\hline $170 \mathrm{~J} / \mathrm{m}^{2}$ & $420 \mathrm{~J} / \mathrm{m}^{2}$ & 1 & $4,7 \mathrm{MPa}$ & $27 \mathrm{MPa}$ \\
\hline
\end{tabular}

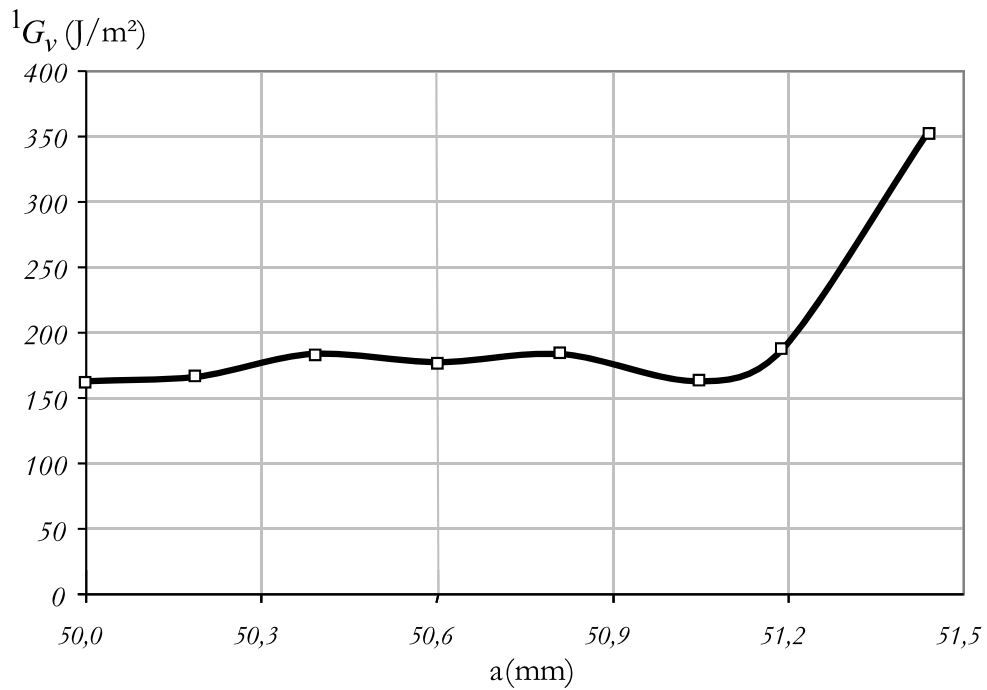

Fig. 5. Evolution du taux de restitution d'énergie de mode I vs. la longueur de fissure.

Fig. 5. Evolution of the open mode part of the energy release rate evolution vs. crack length.

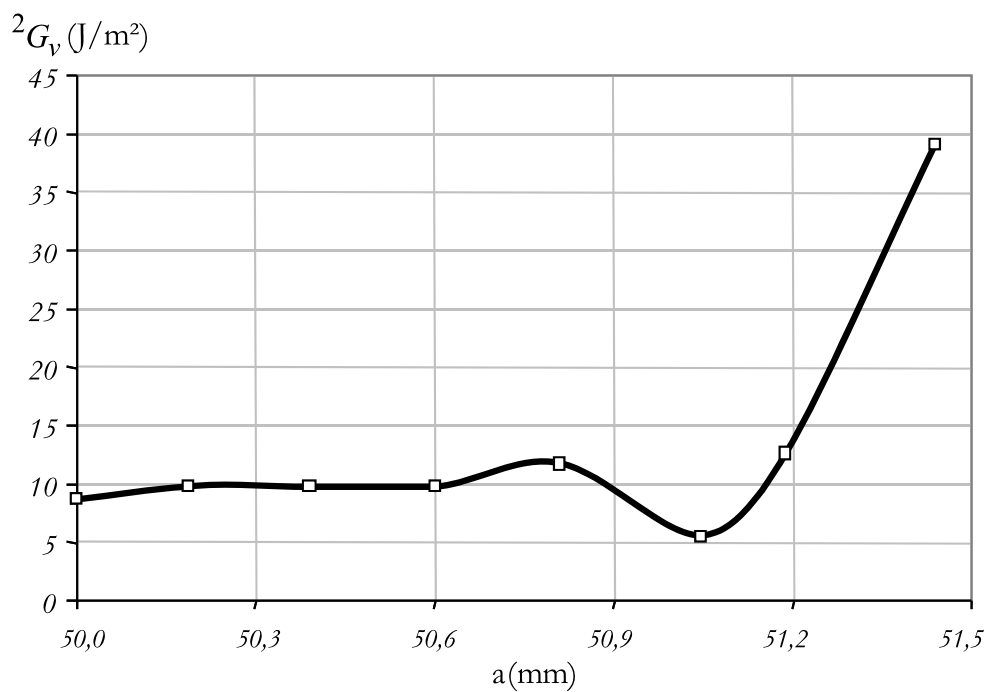

Fig. 6. Evolution du taux de restitution d'énergie de mode II vs. la longueur de fissure.

Fig. 6. Evolution of the shear mode part of the energy release rate evolution vs. crack length.

Les résultats de la simulation sont donnés en termes de partition du taux de restitution d'énergie en mode I et en mode II, Figs. 5 et 6 . Le profil d'évolution des taux de restitution d'énergie met en évidence une plage de stabilité d'environ $1 \mathrm{~cm}$. Le même type de modélisation a été mené en modes I et II pures. Les plages de stabilité observées 
étaient largement plus importantes mais pas pour des longueurs de fissures équivalentes. Le mode mixte représente finalement un compromis entre les deux modes de fissuration.

Il est souvent admis dans les travaux relatifs à la mécanique de la rupture du bois que le mode II est toujours négligeable du fait que la valeur critique ${ }^{2} G_{c}$ est très supérieure à celle relative au mode $\mathrm{I},{ }^{1} G_{c}$. Egalement, la Fig. 6 met en évidence des valeurs faibles en taux de restitution d'énergie. Cependant, nous observons dans ce travail que la mixité va influencer de façon non négligeable les zones de stabilité de la propagation de fissure.

\section{Conclusions}

Il a été proposé une modélisation complète de la propagation de fissure dans un matériau viscoélastique orthotrope. Ce travail repose sur le couplage entre un algorithme complexe traitant, à la fois, de la résolution par éléments finis d'un comportement viscoélastique associé à une progression géométrique du front de fissure, et des outils de la mécanique de la rupture permettant un découplage des modes de rupture lors de chargement complexes induisant du mode mixte. Ce couplage fait intervenir une définition originale de la taille de la zone d'élaboration en mode mixte et l'utilisation d'une fonctionnelle permettant de redéfinir un critère unique d'initiation et de propagation de fissure. Une résolution par éléments finis couplée à un algorithme employant une dichotomie permet de déterminer avec précision les temps de propagation et donc la vitesse de propagation de la fissure. Si l'application repose sur une sollicitation de fluage, cet outil peut être utilisé pour des histoires de chargement plus complexe comme des rampes à différentes vitesses ou des sollicitations de fatigue en force ou en déplacement.

En outre, ce travail s'appuie sur une hypothèse de fracture fragile. Or, il est admis que le matériau bois a une cinétique de fissuration quasi-fragile caractérisée par un endommagement d'une petite zone entourant la pointe de fissure. La propagation de fissure dans ce matériau est également le siège d'un pontage de fibres en arrière du front de fissure. Si ce modèle, à travers son algorithme de propagation de fissure, permet de simuler la décohésion progressive dans la zone endommagée, il ne permet pas de prendre en compte le phénomène de pontage ni l'endommagement en pointe de fissure avant amorçage. Cependant, il existe aujourd'hui des modèles de fissures cohésives qui permettent une relative bonne représentation de ce problème. Ces formalismes sont cependant limités à un comportement élastique et pilotés par des approches en contraintes. Le cas de la viscoélasticité est plus complexe car faisant intervenir des lois énergétiques. Ce travail doit donc être complété pour prendre en compte ce phénomène. Enfin, il reste à valider cette approche par l'expérience. Le phénomène de décohésion doit être validé et visualisé. Il peut être envisagé des techniques de mesure par analyse d'images par corrélation afin d'observer les perturbations du champ de déformation dues aux pontages et à la zone endommagée en font de fissure.

\section{Références}

[1] M. Chaplain, G. Valentin, Fracture mechanics models applied to delayed failure of LVL beams, Holz Roh Werkst. 65 (2008) 7-16.

[2] J.L. Coureau, S. Morel, P.J. Gustafsson, C. Lespine, Influence of the fracture softening behaviour of wood on load-COD curve and $R$-curve, Mater. Struct. 40 (2006) 97-106.

[3] S. Morel, N. Dourado, G. Valentin, Wood: A quasibrittle material $R$-curve behavior and peak load evaluation, Int. J. Fract. 131 (2005) $385-400$.

[4] R. Moutou Pitti, F. Dubois, C. Petit, N. Sauvat, O. Pop, A new $M$-integral parameter for mixed-mode crack growth in orthotropic viscoelastic material, Eng. Fract. Mech. 75 (2008) 4450-4465.

[5] R. Moutou Pitti, Découplage des modes mixtes de rupture dans les matériaux viscoélastiques orthotropes : Modélisation et expérimentation, Thèse de Doctorat, Université de Limoges, http://www.unilim.fr/theses/2008/sciences/2008limo4025/notice.htm, 2008.

[6] R. Moutou Pitti, F. Dubois, P. Octavian, On a specimen providing stable mixed mode crack growth in wood material, C. R. Mecanique 336 (2008) 744-749.

[7] R. Moutou Pitti, F. Dubois, P. Octavian, Mixed-mode fracture in viscoelastic material, in: 12nd ICTAM, Adelaïde, ISBN 978-0-9805142-0-9, 2008.

[8] F.H.K. Chen, R.T. Shield, Conservation laws in elasticity of the $J$-integral type, J. Appl. Mech. Phys. 28 (1977) 1-22.

[9] P.H. Destuynder, M. Djaoua, S. Lescure, Quelques remarques sur la mécanique de la rupture élastique, J. Mec. Theor. Appl. 2 (1983) 113-135.

[10] F. Dubois, C. Chazal, C. Petit, Viscoelastic crack growth process in wood timbers: An approach by the finite element method for mode I fracture, Int. J. Fract. 113 (2002) 367-388.

[11] R.A. Schapery, Correspondence principles and a generalized $J$ integral for large deformation and fracture analysis of viscoelastic media, Int. J. Fract. 25 (1984) 195-223.

[12] G. Valentin, P. Caumes, Crack propagation in mixed mode in wood: A new specimen, Wood Sci. Technol. 23 (1989) $43-53$. 